\title{
CONTINUUM PERCOLATION AND EUCLIDEAN MINIMAL SPANNING TREES IN HIGH DIMENSIONS
}

\author{
By Mathew D. Penrose
}

\section{University of Durham}

\begin{abstract}
We prove that for continuum percolation in $\mathbb{R}^{d}$, parametrized by the mean number $y$ of points connected to the origin, as $d \rightarrow \infty$ with $y$ fixed the distribution of the number of points in the cluster at the origin converges to that of the total number of progeny of a branching process with a Poisson $(y)$ offspring distribution. We also prove that for sufficiently large $d$ the critical points for the existence of infinite occupied and vacant regions are distinct. Our results resolve conjectures made by Avram and Bertsimas in connection with their formula for the growth rate of the length of the Euclidean minimal spanning tree on $n$ independent uniformly distributed points in $d$ dimensions as $n \rightarrow \infty$.
\end{abstract}

1. Introduction. In the simplest Boolean model of continuum percolation, balls of unit diameter are centered at the points of a homogeneous $d$ dimensional Poisson process, and one studies the connected components of the union of the balls. Equivalently, one studies the components of the graph on the Poisson points obtained by connecting each pair of points separated by a distance less than 1 . This and related models have been studied in the contexts of geometric probability, statistics and physics. For a survey, see Meester and Roy [14]; also Alexander [2], Grimmett [9], Hall [10] and Penrose [17].

For $y>0$, let $\mathscr{P}_{y}=\mathscr{P}_{y}(d)$ be a homogeneous Poisson process of rate $y / c_{d}$ on $\mathbb{R}^{d}$, with an extra point inserted at 0 , where $c_{d}=\pi^{d / 2} / \Gamma((d / 2)+1)$ is the volume of the ball of unit radius in dimension $d$. The added point at the origin represents a "typical point" of the Poisson process. Let the 1-graph on $\mathscr{P}_{y}$ be the graph with vertices at each point of $\mathscr{P}_{y}$, and edges between each pair of points $X, Y$ of $\mathscr{P}_{y}$ such that $|X-Y|<1$, where $|\cdot|$ is the Euclidean norm. The parameter $y$ is the mean degree of the vertex at 0 in this graph.

Let $C_{y}=C_{y}(d)$ denote the set of points of $\mathscr{P}_{y}$ in the component containing 0 of the 1-graph on $\mathscr{P}_{y}$; this is analogous to the cluster at the origin in lattice percolation. Let $\left|C_{y}\right|$ denote the cardinality of $C_{y}$, a random variable taking values in $\{1,2,3, \ldots\} \cup\{\infty\}$. Set

$$
f_{k}^{(d)}(y):=P\left[\left|C_{y}(d)\right|=k\right], \quad k=1,2,3, \ldots, \infty .
$$

Here the symbol $:=$ denotes definition. Informally, $f_{k}^{(d)}(y)$ is the probability that a "typical point" of the Poisson process $\mathscr{P}_{y} \backslash\{0\}$ lies in a component of size $k$ of the 1-graph on $\mathscr{P}_{y} \backslash\{0\}$. Thus, $f_{k}^{(d)}(y)$ is a natural object of interest in

Received January 1995; revised December 1995.

AMS 1991 subject classifications. Primary 60K35, 60D05; secondary 60J80, 82B43.

Key words and phrases. Geometric probability, continuum percolation, phase transitions, minimal spanning tree constant, high dimensions, Poisson process, branching process. 
continuum percolation; see, for example, [15], [3] and (11) below. For $k<\infty$, there is an explicit formula for $f_{k}^{(d)}(y)$ [see [15], Proposition 1(a), or [3], (7)], in terms of a rather complicated $k$-fold integral; there is no known closed-form formula for $f_{\infty}^{(d)}(y)$. In this paper we derive simple limiting expressions for these probabilities when the dimension becomes large.

Let $T_{y}$ denote the total number of progeny from a single ancestor of a Galton-Watson branching process with Poisson $(y)$ offspring distribution. In other words, set $T_{y}=\sum_{n=0}^{\infty} Z_{n}$, where $\left(Z_{n}\right)$ is the Galton-Watson process with generating function $G_{y}(s)=\exp (y(s-1))$, with $Z_{0}=1$. Our main results are that $\left|C_{y}\right|$ is stochastically dominated by $T_{y}$, but that the (possibly defective) distribution of $\left|C_{y}(d)\right|$ converges weakly to that of $T_{y}$ as $d \rightarrow \infty$.

A consequence of our results is the resolution of some conjectures made by Avram and Bertsimas [3], who were interested in the distributions of $\left|C_{y}\right|$ and of $T_{y}$ in connection with the series expansions they developed for the growth rates, as $n$ becomes large, of certain random minimal spanning trees on $n$ points, which we describe in detail in Section 3. Our results help to clarify the relationship between these growth rates.

Another consequence of our results is a proof that, for the Boolean model described above in sufficiently high dimensions, there is a range of values of $y$ for which unbounded occupied and vacant regions coexist, partially resolving a conjecture in [14]. This is explained in Section 4.

In Section 5 we describe a sequential construction of a set equivalent to $C_{y}$, and give a heuristic explanation of the ideas behind our proofs. In Sections 6 and 7 we finally prove the main results.

2. The main results. Let $\left(f_{k}(y), k \geq 1\right)$ denote the probability function of $T_{y}$; that is, define

$$
f_{k}(y):=P\left[T_{y}=k\right]=\frac{k^{k-2}}{(k-1) !} y^{k-1} e^{-k y}, \quad k=1,2,3, \ldots
$$

The equality in (2) can be verified using the formula of Dwass [8] for the distribution of $T_{y}$; see also Jagers [11], Theorem 2.11.2, and Section 3 below.

Our results may be compared with the conjectures in Avram and Bertsimas [3], page 129. Our $f_{k}^{(d)}(y)$ is written there as $f_{k}^{(E)}(y)$, while our $f_{k}(y)$ is written there as $f_{k}^{(I)}(y)$. Part (b) of our first result confirms Conjecture 1 of [3].

Proposition 1. (a) Let $y>0$. Then, for all $d,\left|C_{y}\right|$ is stochastically dominated by $T_{y}$. That is,

$$
\sum_{j=1}^{k} f_{j}^{(d)}(y) \geq \sum_{j=1}^{k} f_{j}(y) \quad \text { for all } k \in \mathbb{N} .
$$

(b) For $y>0$,

$$
\lim _{d \rightarrow \infty} f_{k}^{(d)}(y)=f_{k}(y) \quad \text { for all } k \in \mathbb{N}
$$


Our main result says that the limit in (4) can be taken inside the infinite sum over $k \geq 1$. Thus, the distribution of $C_{y}(d)$ converges to that of $T_{y}$ as $d \rightarrow \infty$.

THEOREM 1. Let $y>0$. Let $t=\psi(y)$ be the smallest positive solution to the equation $t=\exp (y(t-1))$. Then

$$
\lim _{d \rightarrow \infty} \sum_{j=1}^{\infty} f_{j}^{(d)}(y)=\sum_{j=1}^{\infty} f_{j}(y)=\psi(y) .
$$

The second equality in (5) is simply the classical extinction probability theorem for the Galton-Watson process.

Theorem 1 shows that as $d \rightarrow \infty$ the percolation probability $f_{\infty}^{(d)}$ converges to the survival probabilty $1-\psi(y)$ for the Galton-Watson process with Poisson $(y)$ offspring distribution. Since $\psi(1)=1$ and $\psi(y)<1$ for $y>1$, it is easily deduced, from Proposition 1(a) and Theorem 1, that the critical value $y_{c}(d):=\inf \left\{y: f_{\infty}^{(y)}>0\right\}$, above which percolation can occur, satisfies

$$
\lim _{d \rightarrow \infty} y_{c}(d)=1 .
$$

Equations (5) and (6) are continuum analogues of the results of Kesten [12] for percolation on the nearest-neighbor integer lattice $\mathbb{Z}^{d}$. The problem of evaluating $y_{c}(d)$ arises both physically (see Hall [10], Section 4.7) and in cluster analysis (see Penrose [17]); in the latter application, $d$ may be arbitrarily large.

The next result confirms the second part of Conjecture 2 of [3], and is immediate from Proposition 1(b) and Theorem 1, along with a routine weak convergence argument.

Corollary 1. For each $K \in \mathbb{N}$,

$$
\lim _{d \rightarrow \infty} \sum_{k \geq K} k^{-1} f_{k}^{(d)}(y)=\sum_{k \geq K} k^{-1} f_{k}(y) .
$$

Finally, we show among other things that the first part of Conjecture 2 of [3] is false.

Proposition 2. Let $d \in \mathbb{N}$. For all $y \geq 0, f_{1}^{(d)}(y)=f_{1}(y)$. Also

$$
\sum_{k \geq K} k^{-1} f_{k}^{(d)}(y) \geq \sum_{k \geq K} k^{-1} f_{k}(y), \quad K=1,2,
$$

but, for some $y_{1}>0$,

$$
\sum_{k \geq 3} k^{-1} f_{k}^{(d)}(y)<\sum_{k \geq 3} k^{-1} f_{k}(y), \quad y<y_{1} .
$$


The quantity $\sum_{k \geq 1} k^{-1} f_{k}^{(d)}(y)$ has physical significance as the "free energy," or mean number per unit volume of components of the 1-graph on $\mathscr{P}_{y} \backslash\{0\}$, divided by the intensity $y / c_{d}$; see Hall [10], Section 4.6, and Penrose [15], Theorem 2. Equations (7) and (8) show that this quantity is bounded below by $E\left[T_{y}^{-1}\right]$, but converges to this lower bound as $d \rightarrow \infty$.

In applications, it is often natural to consider the connected components of the union of balls of radius $1 / 2$, centered at the points of $\mathscr{P}_{y}$. Let $V_{y}$ denote the volume of the component including 0 , which is just the union of such balls centered at the points of $C_{y}$. Clearly, $V_{y} \leq 2^{-d} c_{d}\left|C_{y}\right|$; a further result is that the distribution of $\left(2^{d} / c_{d}\right) V_{y}$ converges to that of $T_{y}$, as $d \rightarrow \infty$. This can be proved using (4), (5) and the proof of Lemma 2 below; we omit the details.

3. The relation to minimal spanning trees. Suppose each edge $\{i, j\}$ of the complete graph on $\{1,2, \ldots, n\}$ is assigned a weight $D_{\{i, j\}}=D_{\{j, i\}}$. The minimal spanning tree (MST) is the connected graph $\tau$ on vertices $\{1,2, \ldots, n\}$ which minimizes $\sum_{\{i, j\} \in \tau} D_{\{i, j\}}$. The quantity thus minimized is the total length $L_{n}$ of the MST. Here we are interested in two particular cases where the $D_{\{i, j\}}$ are random.

In the $d$-dimensional Euclidean model, $X_{1}, X_{2}, \ldots, X_{n}$ are independent and uniformly distributed over $[0,1]^{d}$. We set $D_{\{i, j\}}=\left|X_{i}-X_{j}\right|$. For this model, the total length $L_{n}$ is known to be almost surely asymptotic to $\beta n^{(d-1) / d}$, where $\beta=\beta(d)$ is a positive constant that has not been determined exactly. See Steele [18]; the method of that paper shows that the mean total length of the tree is also asymptotic to $\beta n^{(d-1) / d}$.

In the independent model with parameters $d>0$ and $c>0$, the variables $D_{\{i, j\}}, 1 \leq i<j \leq n$, are mutually independent, positive random variables with common distribution function $(F(x), x \geq 0)$ satisfying

$$
F(x)=c x^{d}(1+o(1)) \quad \text { as } x \rightarrow 0 .
$$

In this model, the mean total length of the MST is again asymptotic to $\beta n^{(d-1) / d}$, but now with a different (known) constant which we denote $\beta=\beta^{(I)}(d, c)$. The superscript $I$ stands for "independent."

Progress in the evaluation of the Euclidean constant $\beta(d)$, and in unifying the two models, has been made by Avram and Bertsimas [3]. They derived closely related series expansions for the constants $\beta(d)$ and $\beta^{(I)}(d, c)$, which we now describe.

For $z>0$, let $P_{k, n}^{(d)}(z)$ [respectively, $P_{k, n}^{(d, c)}(z)$ ] denote the probability that if one removes all edges with $D_{\{i, j\}}>z$ from the complete graph on $n$ points, a specified point lies in a component with exactly $k$ vertices, for the $d$-dimensional Euclidean model (respectively, the independent model with parameters $d$ and $c$ ). Then, for fixed $d$,

$$
P_{k, n}^{(d)}\left[\left(\frac{y}{n c_{d}}\right)^{1 / d}\right] \rightarrow f_{k}^{(d)}(y) \quad \text { as } n \rightarrow \infty,
$$


where $c_{d}$ is the volume of the unit ball and $f_{k}^{(d)}$ is given by (1). For fixed $d$ and $c$,

$$
P_{k, n}^{(d, c)}\left[\left(\frac{y}{n c}\right)^{1 / d}\right] \rightarrow f_{k}(y) \text { as } n \rightarrow \infty,
$$

with $f_{k}$ given by (2). These limits are intuitively clear from the relevant Poisson limit theorems; for explicit proofs, see [3]. For convenience, we restate the main result in [3] [note that (1) in Theorem 1 of that paper has a factor of $k^{-1}$ missing from the right-hand side].

THEOREM 2 (Avram and Bertsimas [3]). In the Euclidean model in dimension $d \geq 2$,

$$
\beta(d)=\lim _{n \rightarrow \infty} \frac{E\left[L_{n}\right]}{n^{(d-1) / d}}=\left(d c_{d}^{1 / d}\right)^{-1} \int_{0}^{\infty} \sum_{k=1}^{\infty}\left(k^{-1} f_{k}^{(d)}(y)\right) y^{(1 / d)-1} d y,
$$

while in the independent model with parameters $d>0$ and $c>0$,

$$
\beta^{(I)}(d, c)=\lim _{n \rightarrow \infty} \frac{E\left[L_{n}\right]}{n^{(d-1) / d}}=\left(d c^{1 / d}\right)^{-1} \int_{0}^{\infty} \sum_{k=1}^{\infty}\left(k^{-1} f_{k}(y)\right) y^{(1 / d)-1} d y
$$

From this result and Proposition 2 above, it follows that $\beta(d) \geq \beta^{(I)}\left(d, c_{d}\right)$. This too was conjectured in [3]. The corollary (7) to Theorem 1 implies that, for each $y$, for large $d$ the integrand in (12) is close to that in (13); it is possible that, as $d \rightarrow \infty, \beta(d)$ is asymptotic to $\beta^{(I)}\left(d, c_{d}\right)$. We do not have a proof, but see Bertsimas and van Ryzin [4] for an asymptotic expression for $\beta(d)$ as $d \rightarrow \infty$.

4. Coexistence in the Boolean model. In this section we consider the Boolean model, which we shall denote $\mathscr{B}_{y}$, given by the union of balls of diameter 1 centered at the points of $\mathscr{P}_{y} \backslash\{0\}$. We call the connected components of $\mathscr{B}_{y}$ the occupied regions, and the components of $\mathbb{R}^{d} \backslash \mathscr{B}_{y}$ the vacant regions. Let $\mathscr{O}_{y}$ (respectively, $\mathscr{V}_{y}$ ) denote the occupied region containing 0 (respectively, the vacant region containing 0 ), so that just one of $\mathscr{O}_{y}$ and $\mathscr{V}_{y}$ is nonempty. Define the following critical points, at which phase transitions occur for this model:

$$
\begin{aligned}
& y_{c}=y_{c}(d)=\inf \left\{y: P\left[\mathscr{O}_{y} \text { is unbounded }\right]>0\right\}, \\
& y_{c}^{*}=y_{c}^{*}(d)=\sup \left\{y: P\left[\mathscr{V}_{y} \text { is unbounded }\right]>0\right\} .
\end{aligned}
$$

It is easily seen that the definition of $y_{c}(d)$ in (14) is consistent with that given just before (6) above.

The number of infinite occupied regions is almost surely 1 if $y>y_{c}$, and a.s. 0 if $y<y_{c}$. The number of infinite vacant regions is a.s. 1 if $y<y_{c}^{*}$ and a.s. 0 if $y>y_{c}^{*}$. Also, $y_{c}=y_{c}^{*}$ for $d=2$, and

$$
y_{c} \leq y_{c}^{*} \text { if } d \geq 3 .
$$

All these results can be found in [14], except for (15), which seems to be new. 
We sketch a proof of (15) for $d=3$ (the proof for other values of $d$ is similar). Let $T$ denote the plane $\left\{\left(x_{1}, x_{2}, x_{3}\right) \subset \mathbb{R}^{3}: x_{3}=0\right\}$. Then $\mathscr{B}_{n} \cap T$ is a Boolean model consisting of a collection of disks, centered at the points of a two-dimensional Poisson process of rate $y / c_{3}$, and with independent identically distributed radii whose common distribution is that of $\left((1 / 4)-U^{2}\right)^{1 / 2}$, where $U$ is uniform on the interval $(-1 / 2,1 / 2)$. If (15) were false, then for $y$ in the interval $\left(y_{c}^{*}, y_{c}\right)$, both $\mathscr{O}_{n} \cap T$ and $\mathscr{V}_{n} \cap T$ would a.s. have no infinite component. But this cannot happen because of known results for the twodimensional Boolean model ([14], Theorem 4.4).

It is conjectured in [14], Chapter 4, that the inequality (15) is strict, which would imply that for an interval of values of $y$ there coexist a.s. an unbounded occupied region and an unbounded vacant region. This is believed to be true, both because of the outcome of simulation studies and because of analogous known results for lattice percolation [6]. We can confirm that the conjecture is true, at least for sufficiently large values of $d$.

THEOREM 3. (a) Let $p_{c}$ be the critical probability for Bernoulli site percolation on the nearest-neighbor lattice $\mathbb{Z}^{2}$. Then, for $d \geq 2$,

$$
y_{c}^{*}(d) \geq 2^{d-2} \log \left(\frac{1}{p_{c}}\right) \pi^{1 / 2}\left(\frac{\Gamma((d+1) / 2)}{\Gamma((d+2) / 2)}\right) .
$$

Consequently, there exists $d_{0} \in \mathbb{N}$ such that $y_{c}^{*}>y_{c}$ for $d \geq d_{0}$.

Proof. For each $(i, j) \in \mathbb{Z}^{2}$, let $\Gamma_{i, j} \subset \mathbb{R}^{d}$ be the cube of side 1 centered at $(i, j, 0, \ldots, 0)$. For each $(i, j) \in \mathbb{Z}^{2}$, we define two perpendicular cylinders $U_{i, j}$ and $V_{i, j}$ contained in $\Gamma_{i, j}$. Let $U_{0,0}$ (respectively $V_{0,0}$ ) be the cylinder of radius $\frac{1}{2}$ and height 1 , with its axis given by the line segment from $\left(-\frac{1}{2}, 0,0, \ldots, 0\right)$ to $\left(\frac{1}{2}, 0,0, \ldots, 0\right)$ [respectively, from $\left(0,-\frac{1}{2}, 0, \ldots, 0\right)$ to $\left.\left(0, \frac{1}{2}, 0, \ldots, 0\right)\right]$. In other words, set

$$
\begin{aligned}
& U_{0,0}=\left\{x=\left(x_{1}, \ldots, x_{d}\right) \in \Gamma_{0,0}: \sum_{i=2}^{d} x_{i}^{2} \leq \frac{1}{4}\right\}, \\
& V_{0,0}=\left\{x=\left(x_{1}, \ldots, x_{d}\right) \in \Gamma_{0,0}: x_{1}^{2}+\sum_{i=3}^{d} x_{i}^{2} \leq \frac{1}{4}\right\} .
\end{aligned}
$$

Using the notation of (17) below, let $U_{i, j}=(i, j, 0, \ldots, 0)+U_{0,0}$, and let $V_{i, j}=(i, j, 0, \ldots, 0)+V_{0,0}$.

We say $(i, j) \in \mathbb{Z}^{2}$ is good if $U_{i, j} \cup V_{i, j}$ contains no points of $\mathscr{P}_{y} \backslash\{0\}$. If $(i, j)$ is good, then the straight-line segment from $\left(i-\frac{1}{2}, j, 0, \ldots, 0\right)$ to $(i+$ $\left.\frac{1}{2}, j, 0, \ldots, 0\right)$ is contained in a vacant region, as is the line segment from $(i, j-$ $\left.\frac{1}{2}, 0, \ldots, 0\right)$ to $\left(i, j+\frac{1}{2}, 0, \ldots, 0\right)$. Therefore, if there is an infinite connected set of good sites $(i, j)$ in $\mathbb{Z}^{2}$, there is an infinite connected vacant region.

Since the volume of $U_{i, j} \cup V_{i, j}$ is less than $2\left(2^{-(d-1)} c_{d-1}\right)$,

$$
P[(i, j) \text { is good }] \geq \exp \left(-2^{2-d}\left(c_{d-1} / c_{d}\right) y\right) .
$$


Therefore, by a comparison with independent Bernoulli site percolation on $\mathbb{Z}^{2}$,

$$
y \leq y_{c}^{*} \quad \text { if } \exp \left(-2^{2-d}\left(c_{d-1} / c_{d}\right) y\right)>p_{c},
$$

so that $y_{c}^{*} \geq 2^{d-2} \log \left(1 / p_{c}\right)\left(c_{d} / c_{d-1}\right)$, which yields (16).

It follows from (16) that $y_{c}^{*}(d) \rightarrow \infty$ as $d \rightarrow \infty$. A comparison with (6) shows that $y_{c}(d)<y_{c}^{*}(d)$ for sufficiently large $d$.

5. Sequential construction of the cluster at 0 . In this section we describe a random algorithm for generating the cluster $C_{y}$. The algorithm also applies to the following more general setting. A bounded open set $B_{1} \subset \mathbb{R}^{d}$ is specified, symmetric in the sense that $-x \in B_{1}$ for all $x \in B_{1}$. Let the $B_{1^{-}}$graph on the points of $\mathscr{P}_{y}$ be given by putting edges between every $X, Y \in \mathscr{P}_{y}$ with $X-Y \in B_{1}$. Let $C_{B_{1}}$ be the component of this graph including the point 0 , and let $\left|C_{B_{1}}\right|$ be the number of vertices of this component.

For $x \in \mathbb{R}^{d}$, define the translate

$$
x+B_{1}:=\left\{x+w: w \in B_{1}\right\} .
$$

To generate $C_{B_{1}}$ sequentially, start with the set $\{0\}$. Add the points of $\mathscr{P}_{y}$ in $B_{1}$, say $X_{1}, \ldots, X_{N}$. Next add the points of $\mathscr{P}_{y}$ in $\left(X_{1}+B_{1}\right) \backslash B_{1}$ (a Poisson process), and then the points in $\left(X_{2}+B_{1}\right) \backslash\left(B_{1} \cup\left(X_{1}+B_{1}\right)\right)$, and so on. At each stage we add the points of $\mathscr{P}_{y}$ in a region that is disjoint from those already considered, so by the independence properties of $\mathscr{P}_{y}$, at each stage we add a new Poisson process on the relevant region.

The proofs of the results in Section 2 are based on this procedure. We describe it more formally by the following algorithm, which generates a sequence $\left(F_{n}\right)$ of random finite subsets of $\mathbb{R}^{d}$, with $F_{1} \subset F_{2} \subset F_{3} \subset \cdots$, and a second increasing sequence $G_{1} \subset G_{2} \subset G_{3} \subset \cdots$, with $G_{i} \subset F_{i}$ for each $i$. Set $A_{i}:=\bigcup_{x \in G_{i}}\left(x+B_{1}\right)$, which represents the region of space already considered before stage $i$ (the construction of sets $F_{i+1}$ and $G_{i+1}$ ), and $F_{i}$ represents the set of points of $\mathscr{P}_{y}$ in $A_{i}$.

Initially set $F_{1}=\{0\}$ and $G_{1}=A_{1}=\varnothing$, the empty set. Then at each successive stage $i=1,2,3, \ldots$ of the algorithm, perform the following two operations:

1. Select $x_{i} \in F_{i} \backslash G_{i}$, and set $G_{i+1}=G_{i} \cup\left\{x_{i}\right\}$.

2. Writing $\left|B_{1}\right|$ for the Lebesgue measure of $B_{1}$, let $N_{i}$ be a Poisson variable with mean $\left(y / c_{d}\right)\left|B_{1}\right|$, and let $N_{i}$ points be placed independently and uniformly in $x_{i}+B_{1}$. These points are to be viewed as the offspring of $x_{i}$. Throw away those offspring that lie in $A_{i}$. Let $F_{i+1}$ be the union of the remaining set of offspring [a Poisson process of rate $y / c_{d}$ on $\left(x_{i}+B_{1}\right) \backslash A_{i}$ ] with $F_{i}$.

The algorithm is terminated at stage $i$ if $G_{i}=F_{i}$. In this case, set $F_{j}=F_{i}$ and $G_{j}=G_{i}$ for all $j>i$. In any event, set $F_{\infty}=\bigcup_{i=1}^{\infty} F_{i}$ and $\left|F_{\infty}\right|=$ $\operatorname{card}\left(F_{\infty}\right)$. 
In step 1 of the $i$ th stage, the choice of $x_{i}$ from possibly several candidates is to be given by a deterministic rule, based on the positions and family trees of the candidates. Any such rule will be said to specify a version of the algorithm.

Intuitively, the Poisson process on $\left(x_{i}+B_{1}\right) \backslash A_{i}$ may be viewed as the set of points of $\mathscr{P}_{y}$ in that set, so that the result of stage $i$ is to add the points of $\mathscr{P}_{y}$ in $\left(x_{i}+B_{1}\right) \backslash A_{i}$. Thus, the random set $F_{\infty}$ should have the same distribution as $C_{B_{1}}$ (when finite) or a subset of $C_{B_{1}}$ (when infinite). Therefore, the following result is clear, given sufficient intuition about the Poisson process. A formal proof (not given here) can be effected by a discretization argument; see [13].

Lemma 1. For $k=0,1,2,3, \ldots$ and $k=\infty, P\left[\left|F_{\infty}\right|=k\right]=P\left[\left|C_{B_{1}}\right|=k\right]$.

We shall also require the following modification of the above algorithm. Specify some open set $B_{2}$ with $B_{2} \supset B_{1}$. In step 2 of the algorithm, set $A_{i}^{\prime}:=$ $\bigcup_{x \in F_{i} \backslash\left\{x_{i}\right\}}\left(x+B_{2}\right)$ (so that $A_{i}^{\prime} \supset A_{i}$ ), and throw away all offspring lying in $A_{i}^{\prime}$ (rather than $A_{i}$ ). Let $F_{\infty}^{\prime}$ be the set of points produced by this modified algorithm. This has the distribution of a subset of $C_{B_{1}}$. Indeed, there is a coupling in which $F_{\infty}^{\prime}$ is a subset of the set $F_{\infty}$ produced by a version of the original algorithm, and therefore

$$
P\left[\left|F_{\infty}^{\prime}\right|=\infty\right] \leq P\left[\left|C_{B_{1}}\right|=\infty\right] .
$$

Heuristics. Here is a rough guide to the ideas behind the proofs of Proposition 1 and Theorem 1 . Take $B$ to be the unit ball in the above algorithm, so that the Poisson variables $N_{i}$ each have mean $y$. Assume we use a version of the algorithm in which $x_{i}$ is selected from the earliest available "generation" of points of $F_{i}$. Then an appropriate subsequence of the sequence $F_{1}, F_{2}, \ldots$ would form a simple branching random walk (BRW), if it were not for the necessary throwing away of points, a phenomenon we denote "interference."

The path traced out in $\mathbb{R}^{d}$ by the above BRW consists of edges which in high dimensions are likely to be (1) of length only just smaller than 1, and (2) mutually orthogonal. Therefore, the probability of any point being discarded before generation $k$ vanishes as $d \rightarrow \infty$. For example, each point is likely to be at a distance approximately $\sqrt{2}$ from its grandparent; hence, interference due to grandparents is unlikely since $\sqrt{2}>1$. This argument will give us Proposition 1.

The proof of Theorem 1 is harder since one is required to consider infinitely many generations. We lay out a grid of squares on $\mathbb{R}^{2}$, and project $\mathbb{R}^{d}$ onto $\mathbb{R}^{2}$ in such a way that the projected BRW has successive steps which are approximately standard bivariate normals. Assuming $y>1$, one can fix $k$ and $m$ such that, with high probability for the projected BRW starting from $m$ particles in one of the squares, there are $m$ particles of generation $k$ in each of the neighboring squares. We run a sequence of BRW's indexed by the squares of the grid, starting with the square at the origin, each BRW running for $k$ generations. The zeroth generation of the BRW for square 0 is the single point at 0 , and for each subsequent square the zeroth generation 
of the corresponding BRW consists (if possible) of $m$ points lying "above" that square (in terms of the projection), taken from the $k$ th generation of one of the previous BRW's. The whole collection of BRW's is a sort of pruned BRW starting from the origin.

We say a square is "occupied" if the BRW for the corresponding step of the algorithm produces at least $m$ particles at the $k$ th generation lying above each neighboring square. The probability that the square 0 is occupied is close to $1-\psi(y)$, while other squares have probability close to 1 of being occupied.

To make this algorithm really correspond to a version of the algorithm above for producing $F_{\infty}$, we must throw some of the particles of the BRW's away. The key to the proof is the fact that the probability of interference is small at each step, and therefore, even with interference taken into account, the probability that the algorithm produces an infinite path from 0 is close to $1-\psi(y)$ by a comparison with (oriented) site percolation of occupied squares on the grid.

At each step the BRW runs for only a fixed finite number of generations. By the same argument as for Proposition 1, for large $d$ the chances of interference due to any particular previous step are small. Finally, the chance of interference due to far-away steps can be made 0 , if we truncate the (twodimensional normal) distribution of the steps of the projected random walk at some large range $\rho$; indeed, the projected BRW starting from a given square can then only spread within a range $\rho k$ from that square, and so cannot interfere with any BRW from a distance greater than $2 \rho k$ from that square. If $\rho$ is sufficiently large, the truncation need not invalidate any of the earlier parts of the argument.

\section{Proof of propositions.}

Proof of Proposition 1. In this section and the next, let $B$ be the unit ball $\left\{x \in \mathbb{R}^{d}:|x|<1\right\}$. Let $\left(Z_{n}^{d}, n=0,1,2, \ldots\right)$ be the following BRW in $\mathbb{R}^{d}$. Here $Z_{n}^{d}$ is a random subset of $\mathbb{R}^{d}$. Each element (or "particle") of $Z_{n}^{d}$ is replaced in $Z_{n+1}^{d}$ by a Poisson number of offspring with mean $y$, and the offspring of a particle at $x$ are uniformly distributed over $x+B$. Set $Z_{0}$ to be a single particle at 0 . The distribution of the total progeny $\sum_{n=0}^{\infty} \operatorname{card}\left(Z_{n}^{d}\right)$ is that of $T_{y}$.

Let the particles of this BRW be ordered as follows; all members of an earlier generation precede all members of a later generation, and the members of any particular generation are ranked in order of increasing modulus. Modify the BRW by throwing away any particle $X$ that lies in any translate of $B$ centered at a particle of the BRW that precedes the parent of $X$ in the above ordering. Also throw away all descendants of any particle thus discarded.

It is not hard to see that this construction is equivalent to a version of the algorithm described in Section 5 (with $B_{1}=B$ ). The throwing away of branches of the BRW corresponds to the discarding of offspring in $A_{i}$ at stage $i$ of that algorithm. The construction shows by an explicit coupling that $\left|F_{\infty}\right|$ given by the algorithm is stochastically dominated by $T_{y}$, and therefore, by Lemma 1 , so is $\left|C_{y}\right|$. 
To prove part (b), that $f_{k}^{(d)}(y) \rightarrow f_{k}(y)$ as $d \rightarrow \infty$, it suffices to prove the following lemma.

LEMMA 2. Let $y \geq 0$ and $k \in \mathbb{N}$. Then $\lim _{d \rightarrow \infty}\left(\sum_{j=1}^{k} f_{j}^{(d)}(y)-\sum_{j=1}^{k} f_{j}(y)\right)$
$=0$.

Proof. The quantity inside the limit is the probability that, in the above construction, (i) the BRW has total progeny greater than $k$, and (ii) after throwing away some of the branches in the prescribed manner, we are left with at most $k$ particles. This event is contained in the complement of the event, denoted $E_{k}$, that none of the first $k$ particles in the above ordering of the BRW is thrown away.

Let $W_{i}$ denote the position of the $i$ th particle of the BRW (in the given ordering). Let $E_{i}^{\prime}$ denote the event that (i) none of the offspring of $W_{i}$ lies in the union of the unit balls centered at its predecessors $W_{1}, \ldots, W_{i-1}$; (ii) no two of its offspring are separated by a distance less than 1 ; and (iii) each of its offspring lies outside the Euclidean ball of radius $3 / 4$ centered at $W_{i}$.

Set $F_{k}=\bigcap_{i=1}^{k} E_{i}^{\prime}$. Clearly, $E_{k} \supset F_{k-1}$. The next lemma shows that $P\left[F_{1}\right] \rightarrow$ 1 as $d \rightarrow \infty$, and that, for each $k>1, P\left[F_{k} \mid F_{k-1}\right] \rightarrow 1$ as $d \rightarrow \infty$; therefore, $P\left[E_{k}\right] \rightarrow 1$, which completes the proof.

LemMa 3. Suppose $\mathbf{X}(d)$ and $\mathbf{Y}(d)$ are independent and uniformly distributed on $B$. Then

$$
\lim _{d \rightarrow \infty}\left(\sup \left\{P[|\mathbf{X}(d)-x| \leq 1]: x \in \mathbb{R}^{d},|x| \geq 3 / 4\right\}\right)=0
$$

and

$$
\lim _{d \rightarrow \infty} P[|\mathbf{X}(d)-\mathbf{Y}(d)| \leq 1]=0 .
$$

Proof. The first conclusion (19) is trivial. To prove (20), note that

$$
|\mathbf{X}(d)-x|^{2}=|x|^{2}+|\mathbf{X}(d)|^{2}-2|\mathbf{X}(d) \cdot x| .
$$

By (19), it suffices to prove that $\mathbf{X}(d) \cdot x$ converges to 0 in probability, uniformly on $\{x:(3 / 4) \leq|x| \leq 2\}$. Write $\mathbf{X}(d)$ in coordinates as $\left(X^{1}(d), X^{2}(d), \ldots, X^{d}(d)\right)$. By symmetry, $\mathbf{X}(d) \cdot x$ has the same distribution as $|x| X^{1}(d)$. Again, by symmetry, $E\left[\left|X^{1}(d)\right|^{2}\right] \leq 1 / d$, so $X^{1}(d)$ converges to 0 in $L^{2}$, hence in probability.

Finally, (21) follows at once from (19) and (20). 
Proof of Proposition 2. Clearly, $\left|C_{y}\right|=1$ if and only if there are no points of $\mathscr{P}_{y}$ in the unit ball centered at 0 . Therefore, $f_{1}^{(d)}(y)=\exp (-y)=$ $f_{1}(y)$. The inequality

$$
\sum_{k \geq K} k^{-1} f_{k}^{(d)}(y) \geq \sum_{k \geq K} k^{-1} f_{k}(y)
$$

holds for $K=1$ by the stochastic domination in part (a) of Proposition 1. It then holds also for $K=2$ because $f_{1}^{(d)}(y)=f_{1}(y)$. However, we now show that, for fixed $d$ and small enough $y$, the inequality is false for $K=3$.

As $y \rightarrow 0, f_{3}(y)$ and $f_{3}^{(d)}(y)$ are both $O\left(y^{2}\right)$, whereas $\sum_{k>3} f_{k}(y)$ and $\sum_{k>3} f_{k}^{(d)}(y)$ are $O\left(y^{3}\right)$. Therefore, it suffices to show that there are constants $c_{1}<c_{2}$ such that, as $y \rightarrow 0$,

$$
\sum_{k \geq 3} f_{k}^{(d)}(y) \sim c_{1} y^{2}
$$

while

$$
\sum_{k \geq 3} f_{k}(y) \sim c_{2} y^{2}
$$

Let $N_{1}$ and $N_{2}$ be independent Poisson $(y)$ variables, where $N_{1}$ represents the size of the first generation and $N_{2}$ represents the number of offspring of the (only) member of the first generation in the case where $N_{1}=1$. Then

$$
\begin{aligned}
\sum_{k \geq 3} f_{k}(y) & =P\left[N_{1} \geq 2\right]+P\left[N_{1}=1, N_{2} \geq 1\right] \\
& =\left(e^{-y} y^{2} / 2\right)+e^{-2 y} y^{2}+o\left(y^{2}\right),
\end{aligned}
$$

so that (23) holds with $c_{2}=3 / 2$.

Set $\gamma:=P[|\mathbf{X}+\mathbf{Y}|>1]$, where $\mathbf{X}$ and $\mathbf{Y}$ are independent and uniform on the unit ball. Consider the construction of $F_{\infty}$ via a BRW, as described in the previous section. In this construction, no members of generation 1 will be thrown away (as their parent has no predecessor). However, given that generations 1 and 2 of the BRW are of size 1, the particle in generation 2 is discarded with probability $1-\gamma$. Therefore,

$$
\sum_{k \geq 3} f_{k}^{(d)}(y)=P\left[N_{1} \geq 2\right]+\gamma P\left[N_{1}=1\right] P\left[N_{2}=1\right]+o\left(y^{2}\right),
$$

so that (22) holds with $c_{1}=(1 / 2)+\gamma$.

7. Proof of Theorem 1. Define the linear mapping $L: \mathbb{R}^{d} \rightarrow \mathbb{R}^{2}$ by

$$
L\left(x_{1}, x_{2}, \ldots, x_{d}\right)=d^{1 / 2}\left(x_{1}, x_{2}\right) .
$$

The next result is an extension of the well-known fact that a single coordinate of a uniformly distributed variable on a high-dimensional sphere is approximately normal. 
Lemma 4. Suppose $\mathbf{X}(d)=\left(X_{1}(d), \ldots, X_{d}(d)\right)$ is uniformly distributed over $\left\{x \in \mathbb{R}^{d}:|x|<1\right\}$. Then the two-dimensional random vector $L(\mathbf{X}(d))$ converges in distribution to the bivariate normal distribution, written $N(0, I)$, with mean 0 and the identity matrix I as covariance matrix.

Proof. Let $Z_{i}, i \geq 1$, be independent $N(0,1)$ random variables. For each $d$, set $Y_{i}(d)=d^{-1 / 2} Z_{i}$, set $\mathbf{Y}(d)=\left(Y_{1}(d), Y_{2}(d), \ldots, Y_{d}(d)\right)$ and $R_{d}=$ $|\mathbf{Y}(d)|$. Let $U_{d}$ be an independent random variable, distributed over $(0,1)$ with density $d y^{d-1}, 0<y<1$. By the strong law of large numbers, $R_{d} \rightarrow 1$ as $d \rightarrow \infty$, so that $U_{d} / R_{d}$ converges to 1 in probability.

Set $\mathbf{X}(d)=\left(U_{d} / R_{d}\right) \mathbf{Y}(d)$. Then $\mathbb{X}(d)$ is uniformly distributed over the unit ball. Since $L(\mathbf{Y}(d))$ has the $N(0, I)$ distribution, it follows that $L(\mathbf{X}(d))=$ $\left(U_{d} / R_{d}\right) L(\mathbf{Y}(d)) \rightarrow N(0, I)$ in distribution (see Billingsley [5], page 28, exercise 1$)$.

Proof of Theorem 1. It follows from (3) in Proposition 1 that

$$
\liminf _{d \rightarrow \infty} \sum_{j=1}^{\infty} f_{j}^{(d)}(y) \geq \psi(y) .
$$

Therefore, it suffices to show that, for any $y$ with $\psi(y)<1$ (i.e., $y>1$ ) and any $\varepsilon>0$,

$$
\limsup _{d \rightarrow \infty} \sum_{j=1}^{\infty} f_{j}^{(d)}(y) \leq \psi(y)+3 \varepsilon .
$$

With $\mathbf{X}(d)$ as in Lemma 4 and with $\mathbf{Z} \sim N(0, I)$, the two-dimensional standard normal, for $\rho>0$ define $y_{\rho}(d)$ and $y_{\rho}(\infty)$ by

$$
P[|L(\mathbf{X}(d))|<\rho]=y_{\rho}(d) / y, \quad P[|\mathbf{Z}|<\rho]=y_{\rho}(\infty) / y .
$$

We shall show that $\rho$ can be chosen so that, for large $d$, with probability close to $1-\psi(y)$, there is an infinite path from 0 in the 1 -graph on $\mathscr{P}_{y}$, consisting of Poisson points $Y_{i}$ satisfying $\left|L\left(Y_{i+1}-Y_{i}\right)\right|<\rho$. In other words, we consider the $B^{\rho}$-graph on $\mathscr{P}_{y}$, where we set

$$
B^{\rho}:=\left\{x \in \mathbb{R}^{d}:|x|<1 \text { and }|L(x)|<\rho\right\} .
$$

We shall show there exists $\rho$ such that, for large $d$,

$$
P\left[C_{B^{\rho}} \text { is infinite }\right] \geq 1-\psi(y)-2 \varepsilon,
$$

and (25) will then follow at once.

The argument to prove (28) is similar to that in Penrose [16] and elsewhere, a comparison with oriented percolation on the lattice $\mathscr{L}:=\left\{(i, j) \in \mathbb{Z}^{2}: i \geq\right.$ $0,|j| \leq i,(i+j) / 2 \in \mathbb{Z}\}$, with oriented edges from $(i, j)$ to $(i+1, j \pm 1)$. We can (and do) choose $\delta \in(0, \varepsilon / 3)$ such that, for oriented site percolation on $\mathscr{L}$ with parameter $p \geq 1-5 \delta$, the probability exceeds $1-\varepsilon$ that there is an infinite path from 0 of occupied sites in $\mathscr{L}$. See Durrett [7]. For $(i, j) \in \mathscr{L}$, let $A_{i, j}$ be the closed square of side 1 in $\mathbb{R}^{2}$ centered at $(i, j)$. 
Let $\left(Z_{n}^{d}, n=0,1,2, \ldots\right)$ be the $d$-dimensional branching random walk (BRW) described in Section 6, in which a particle at $x$ has a Poisson $(y)$ number of offspring, uniformly distributed on the unit ball $x+B$. Let $Z_{n}^{d, \rho}$ be the BRW of the same form, except that the number of offspring is Poisson with mean $y_{\rho}(d)$, and the offspring are placed uniformly on $x+B^{\rho}$.

Let $Z_{n}^{\infty}$ be the BRW in $\mathbb{R}^{2}$ with the Poisson $(y)$ offspring distribution, whose steps have the $N(0, I)$ distribution. Let $Z_{n}^{\infty, \rho}$ be the BRW in $\mathbb{R}^{2}$ with the Poisson $\left(y_{\rho}(\infty)\right)$ offspring distribution, whose steps have the distribution of an $N(0, I)$ variable conditioned to lie in the disk of radius $\rho$. For $A \subset \mathbb{R}^{2}$, let $Z_{n}^{\infty}(A)$ denote the number of particles of the $n$th generation of the BRW $\left(Z_{n}^{\infty}\right)$ in $A$, and likewise for $Z_{n}^{\infty, \rho}, Z_{n}^{d}$ and $Z_{n}^{d, \rho}$.

Let $y>1$. By the proof of Lemma 2 of [16] (but see the remark below), there exist $m>0$ and $k>0$ such that, for sufficiently large $d$,

$$
P\left[Z_{k}^{\infty}\left(A_{1,1}\right) \geq m \text { and } Z_{k}^{\infty}\left(A_{1,-1}\right) \geq m\right]>1-\delta \quad \text { if }\left|Z_{0}^{\infty}\left(A_{0,0}\right)\right| \geq m .
$$

The proof of (3.3) of [16] uses the multivariate local limit theorem, but this is not required here because the individual steps of the BRW $\left(Z_{n}^{\infty}\right)$ are already normal, and the distribution of the sum of independent normal variables is known exactly. This was pointed out to the author by R. Meester.

By the proof of Lemma 3 of [16] (again, a slight simplification is possible here), there exists $k_{1}>0$ such that, for sufficiently large $d$,

$$
P\left[Z_{k_{1}}^{\infty}\left(A_{1,1}\right) \geq m \text { and } Z_{k_{1}}^{\infty}\left(A_{1,-1}\right) \geq m\right]>1-\psi(y)-\delta \quad \text { if } Z_{0}^{\infty}=\{0\} .
$$

By an obvious coupling (discard all offspring at a distance greater than $\rho$ from the parent), given the same initial value the point process $Z_{n}^{\infty, \rho}$ converges weakly to $Z_{n}^{\infty}$ as $\rho \rightarrow \infty$, in the sense of, for example, Aldous and Steele [1]. Therefore, it is possible to take $\rho$ to be so big that (29) and (30) are still true with the process $\left(Z_{n}^{\infty}\right)$ replaced by $\left(Z_{n}^{\infty, \rho}\right)$. Similarly, by Lemma 4 we can then find $d_{0}$ such that for $d \geq d_{0}$ they still hold with $\left(Z_{n}^{\infty, \rho}\right)$ replaced by the image under $L$ of $\left(Z_{n}^{d, \rho}\right)$; that is, there exist $\rho$ and $d_{0}$ such that, for $d \geq d_{0}$,

$$
\begin{aligned}
P\left[Z_{k}^{d, \rho}\left(L^{-1}\left(A_{1,1}\right)\right)>m \text { and } Z_{k}^{d, \rho}\left(L^{-1}\left(A_{1,-1}\right)\right)>m\right]>1-\delta, \\
\text { if }\left|Z_{0}^{d, \rho}\left(L^{-1}\left(A_{0,0}\right)\right)\right| \geq m,
\end{aligned}
$$

and

$$
\begin{array}{r}
P\left[Z_{k_{1}}^{d, \rho}\left(L^{-1}\left(A_{1,1}\right)\right)>m \text { and } Z_{k_{1}}^{d, \rho}\left(L^{-1}\left(A_{1,-1}\right)\right)>m\right]>1-\psi(y)-\delta \\
\text { if } Z_{0}^{d, \rho}=\{0\} .
\end{array}
$$

The value of $\rho$ remains fixed for the remainder of the proof.

Choose $k_{2}$ so large that for a Galton-Watson process $\left(Z_{n}\right)$ with generating function $G_{y}$, the total number of progeny in the first $k$ generations (from $m$ ancestors) or in the first $k_{1}$ generations (from one ancestor) is unlikely to 
exceed $k_{2}$, that is, so that

$$
\max \left(P\left[\sum_{n=0}^{k} Z_{n}>k_{2} \mid Z_{0}=m\right], P\left[\sum_{n=0}^{k_{1}} Z_{n}>k_{2} \mid Z_{0}=1\right]\right)<\delta .
$$

We now describe an algorithm which may be viewed as a version of the modified algorithm for generating $F_{\infty}^{\prime}$ in Section 5 , taking $B_{1}$ to be $B^{\rho}$ and $B_{2}$ to be the unit ball $B$. The aim is to show that the algorithm continues forever, so that $\left|F_{\infty}^{\prime}\right|=\infty$, with probability at least $1-\psi(y)-2 \varepsilon$; this will give us (28) by the corollary (18) of Lemma 1 . The algorithm consists of a sequence of steps, indexed by the sites $(i, j)$ of the lattice $\mathscr{L}$. Each step consists of a copy of the BRW $\left(Z_{n}^{d, \rho}\right)$, run for a finite number of generations. The steps are to be performed in the order $(0,0),(1,-1),(1,1),(2,-2),(2,0),(2,2),(3,-3)$, $(3,-1), \ldots$ Step $(0,0)$ is special since here the BRW runs for $k_{1}$ generations and starts from a single point at 0 . In each subsequent step $(i, j)$, the BRW runs for $k$ generations and starts from a set of $m$ points in $L^{-1}\left(A_{i, j}\right)$. Initially, we set each site $(i, j)$ of $\mathscr{L}$ to be "vacant," but we shall change its status to "occupied" if step $(i, j)$ is "successful."

Step $(0,0)$ is to run a BRW $Z_{n}^{d, \rho}, n=0,1, \ldots, k_{1}$, with $Z_{0}^{d, \rho}=\{0\}$. Order the particles of the BRW as follows. Particles of an earlier generation are to precede those of a later one. Within generation $n, n>0$, particles with distinct parents inherit the ordering placed on their parents, while siblings are ordered by modulus.

Now modify this branching process as follows. Consider successively each point $X$ of the BRW after generation 0 (in the ordering given, starting with the point of smallest modulus in $Z_{1}^{d, \rho}$ ), and remove $X$ (along with its descendants) if it lies in any translate of $B$ centered at an (unremoved) particle that precedes $X$ in the ordering, and is not a sibling or parent of $X$. If at the end of this procedure more than $k_{2}$ particles remain, remove all but the first $k_{2}$ of the remaining particles in the ordering. Let the set $F$ consist of all remaining particles in generations $0,1,2, \ldots, k_{1}$. This corresponds to the set $F_{i}$ in the algorithm of Section 5 , at the end of step $(0,0)$, while $G_{i}$ at this point consists of all remaining particles except those in generation $k_{1}$.

Step $(0,0)$ is deemed to be "successful" if (i) $Z_{k_{1}}^{d, \rho}\left(L^{-1}\left(A_{1,1}\right)\right) \geq m$ and $Z_{k_{1}}^{d, \rho}\left(L^{-1}\left(A_{1,-1}\right)\right) \geq m$; (ii) no particle has cause to be removed; and (iii) no particle of generation $k_{1}$ lies within a distance less than $3 / 4$ of its parent or siblings. If step $(0,0)$ is successful, change the status of site $(0,0)$ to "occupied." If it is unsuccessful, the algorithm is terminated.

Assuming step $(0,0)$ to have been successful, proceed with step $(1,-1)$ as follows. Let the set $S_{1,-1}$ consist of the $m$ particles in $L^{-1}\left(A_{1,-1}\right)$ of the $k_{1}$ th generation of step $(0,0)$ with smallest modulus. Such a set exists by condition (i) for the success of step $(0,0)$. Run another BRW, this time of $k$ generations, again denoted $\left(Z_{n}^{d, \rho}\right)$, this time with $Z_{0}^{d, \rho}=S_{1,-1}$. Again, order the particles of the new BRW by the same rule as in step $(0,0)$. Consider successively each particle $X$ after generation 0 of the new BRW, in the ordering given, and 
remove $X$ (along with its descendants) if it lies in a translate of $B$ centered at any unremoved particle of the BRW preceding $X$ in the ordering, other than its parent and siblings, or at any point of the set $F$ created in step $(0,0)$, other than the parent of $X$. Then remove all but the first $k_{2}$ of the remaining particles of this new BRW. Add all the remaining particles of this new BRW to the set $F$. The new set $F$ corresponds to the set $F_{i}$ in the algorithm of Section 5 , at the end of step $(1,-1)$.

Step $(1,-1)$ is deemed to be a success if, for this BRW, (i) $Z_{k}^{d, \rho}\left(L^{-1}\left(A_{2,0}\right)\right) \geq$ $m$ and $Z_{k}^{d, \rho}\left(L^{-1}\left(A_{2,-2}\right)\right) \geq m$; (ii) no particle of this BRW has cause to be removed; and (iii) no particle of generation $k$ lies within a distance $3 / 4$ of its parent or siblings. If this step is a success, change the status of $(1,-1)$ to "occupied."

Now perform step $(1,1)$, and continue in the same manner. At each stage, pick the next site $(i, j)$ in the ordering of $\mathscr{L}$ which has not already been considered and for which there is an oriented path of occupied sites in $\mathscr{L}$ from $(0,0)$ to $(i-1, j-1)$ [which we call event $\left.E_{-}(i, j)\right]$, or to $(i-1, j+1)$ [which we call event $E_{+}(i, j)$ ]. If no such site $(i, j)$ exists, the algorithm terminates. For any $(i, j)$ having been thus picked, if event $E_{+}(i, j)$ has occurred let $S_{i, j}$ consist of the $m$ particles in $L^{-1}\left(A_{i, j}\right)$ with smallest modulus out of those in generation $k$ (or $k_{1}$ in the case $i=1$ ) of the BRW of step $(i-1, j+1)$. If event $E_{-}(i, j)$ has occurred but $E_{+}(i, j)$ has not, let $S_{i, j}$ be defined similarly using the BRW of step $(i-1, j-1)$ instead of $(i-1, j+1)$.

Then in step $(i, j)$, run a new BRW, again denoted $\left(Z_{n}^{d, \rho}\right)$, for $k$ generations as before, now with $Z_{0}^{d, \rho}=S_{i, j}$. Again order the particles of the new BRW as in the case of step $(0,0)$, and consider successively each particle $X$ subsequent to generation 0 , removing $X$ (along with its descendants) if it lies in the translate of $B$ centered at any unremoved particle of the BRW preceding $X$ in the ordering, other than its parent or siblings, or at any particle of the set $F$ existing at the start of step $(i, j)$, other than the parent of $X$. Then remove all but the first $k_{2}$ of the remaining particles of the BRW, and add the remaining particles to the set $F$.

Step $(i, j)$ is "successful" if (i) it produces at least $m$ sites of the $k$ th generation in $L^{-1}\left(A_{i+1, j+1}\right)$ and at least $m$ sites of the $k$ th generation in $L^{-1}\left(A_{i+1, j-1}\right)$ and if it satisfies conditions (ii) and (iii) described for step $(1,-1)$ above. If step $(i, j)$ is "successful," the status of site $(i, j)$ is changed to "occupied."

The resulting sequence of BRW's is equivalent to a version of the algorithm for generating $F_{\infty}^{\prime}$ described in Section 5. If the algorithm never terminates, then $F_{\infty}^{\prime}$ is infinite.

For sufficiently large $d$, the probability that step $(0,0)$ is successful exceeds $1-\psi(y)-3 \delta$, by (32), (33) and Lemma 3 . We now show that if $d$ is sufficiently large, then, for each $(i, j)$ other than $(0,0)$ in $\mathscr{L}$, given that $(i, j)$ is picked at all, the probability that step $(i, j)$ satisfies conditions (i)-(iii) to be "successful" exceeds $1-5 \delta$.

Condition (ii) fails if (a) the total progeny of the $k$-generation BRW of step $(i, j)$ exceeds $k_{2}$, or (b) one of the particles in the BRW of step $(i, j)$ lies in 
the translate of $B$ centered at one of its predecessors (other than its parent or siblings) in that BRW, or (c) one of the particles in the BRW of step $(i, j)$ lies in a translate of $B$ centered at a particle already in $F$ as a result an earlier step of the algorithm. By (33) and Lemma 3,

$$
P[\text { (ii) fails by (a) or (b) in step }(i, j)]<2 \delta \text {. }
$$

If condition (ii) fails by (c), one of the first $k_{2}$ particles in the BRW of step $(i, j)$ lies in a translate of $B$ centered at a particle created at an earlier step of the algorithm, say step $\left(i^{\prime}, j^{\prime}\right)$. Since the steps of the projected BRW $\left(L\left(Z_{n}^{d, \rho}\right)\right)$ are of length at most $\rho$, it is impossible for this to happen unless $|x-y| \leq\left(2 k+k_{1}\right) \rho$, for some $x \in A_{i, j}$ and $y \in A_{i^{\prime}, j^{\prime}}$. The number of such $\left(i^{\prime}, j^{\prime}\right)$ is bounded above by some constant $K$, independent of $i, j$ and $d$, and, for each such $\left(i^{\prime}, j^{\prime}\right)$, at most $k_{2}$ sites are added to $F$. Using the fact that the previous successful step $(i-1, j+1)$ or $(i-1, j-1)$ satisfies conditions (ii) and (iii), we have

$$
P[\text { (ii) fails by (c) only, in step }(i, j)] \leq k_{2}^{2} K \sup _{|x| \geq 3 / 4}\{P[|\mathbf{X}-x| \leq 1]\},
$$

where $\mathbf{X}$ is uniform on $B^{\rho}$. By Lemma 3 , for sufficiently large $d$, this upper bound is at most $\delta$. Also, by (31), for large enough $d$ we have

$$
P[\text { (ii) holds but (i) fails in step }(i, j)] \leq \delta,
$$

and by Lemma 3, for large $d$,

$$
P[\text { (iii) fails in } \operatorname{step}(i, j)] \leq \delta .
$$

It follows from (34)-(37) that for sufficiently large $d$ the probability that step $(i, j)$ is successful exceeds $1-5 \delta$. A comparison with oriented percolation with parameter $1-5 \delta$ shows that the branching random walk algorithm continues forever, with probability at least $(1-\psi(y)-3 \delta)(1-\varepsilon)$, the first factor being the lower bound on the probability of success in step $(0,0)$. Then $(28)$ follows by Lemma 1 and the choice of $\delta$.

Acknowledgments. I thank Mike Steele for drawing to my attention the conjectures of Avram and Bertsimas; also Ronald Meester and Anish Sarkar, for some useful comments and questions.

\section{REFERENCES}

[1] Aldous, D. and SteEle, J. M. (1992). Asymptotics for Euclidean minimal spanning trees on random points. Probab. Theory Related Fields 92 247-258.

[2] AleXander, K. (1993). Finite clusters in high-density continuous percolation: compression and sphericality. Probab. Theory Related Fields 97 35-63.

[3] Avram, F. and Bertsimas, D. (1992). The minimum spanning tree constant in geometrical probability and under the independent model: a unified approach. Ann. Appl. Probab. 2 113-130.

[4] Bertsimas, D. J. and van RYZIN, G. (1990). An asymptotic determination of the minimum spanning tree and minimum matching constants in geometrical probability. Oper. Res. Lett. 9 223-231. 
[5] Billingsley, P. (1968). Convergence of Probability Measures. Wiley, New York.

[6] Campanino, M. and Russo, L. (1985). An upper bound on the critical percolation probability for the three-dimensional cubic lattice. Ann. Probab. 13 478-491.

[7] Durrett, R. (1988). Lecture Notes on Particle Systems and Percolation. Wadsworth and Brooks-Cole, Pacific Grove, CA.

[8] Dwass, M. (1969). The total progeny in a branching process and a related random walk. J. Appl. Probab. 6 682-686.

[9] Grimmett, G. (1989). Percolation. Springer, New York.

[10] Hall, P. (1988). Introduction to Coverage Processes. Wiley, New York.

[11] JAGERs, P. (1975). Branching Processes with Biological Applications. Wiley, New York.

[12] Kesten, H. (1990). Asymptotics in high dimensions for percolation. In Disorder in Physical Systems: A Volume in Honour of J. M. Hammersley (G. R. Grimmett and D. J. A. Welsh, eds.). Clarendon, Oxford.

[13] Meester, R., Penrose, M. D. and Sarkar, A. (1995). The random connection model in high dimensions. Preprint.

[14] Meester, R. and Roy, R. (1996). Continuum Percolation. Cambridge Univ. Press.

[15] Penrose, M. D. (1991). On a continuum percolation model. Adv. in Appl. Probab. 23 536556.

[16] Penrose, M. D. (1993). On the spread-out limit for bond and continuum percolation. Ann. Appl. Probab. 3 253-256.

[17] Penrose, M. D. (1995). Single linkage clustering and continuum percolation. J. Multivariate Anal. 52 94-109.

[18] Steele, J. M. (1988). Growth rates of Euclidean minimal spanning trees with power weighted edges. Ann. Probab. 16 1767-1787.

Department of Mathematical Sciences

UNIVERSITY OF DURHAM

SOUTH ROAD

DURHAM DH1 3LE

UNITED KINGDOM

E-MAIL: mathew.penrose@durham.ac.uk 